Harold A. McAlister and William I. Hartikopf (eds.)

\title{
CORAVEL Surveys to Study Binaries of Different Masses and Ages
}

\author{
M. MAYOR \& A. DUQUENNOY \\ Geneva Observatory, 51 Chemin des Maillettes, CH-1290 Sauverny, \\ Switzerland
}

\section{J.-L. HALBWACHS}

Strasbourg Observatory, 11 rue de l'Université, F-69000 Strasbourg, France

\section{J.-C. MERMILLIOD}

Lausanne Astronomical Institute, CH-1290 Chavannes-des-Bois, Switzerland

\begin{abstract}
Preliminary results are given for a systematic survey of K-dwarf stars in the solar vicinity. Nine companions to the $G$ and $K$ dwarfs have very low $\mathcal{M}_{2} \sin i$, less than $0.08 \mathcal{M}_{\odot}$. These detections from a sample of $540 \mathrm{G}$ and $\mathrm{K}$ primary stars support the reality of the existence of companions with mass below $0.08 \mathcal{M}_{\odot}$ : brown dwarfs exist.

A comparison of the relative mass function distribution $f(m) / \mathcal{M}_{1}$ between lowmass $\left(\mathcal{M}_{1}<1.3 \mathcal{M}_{\odot}\right)$ and intermediate-mass $\left(2<\mathcal{M}_{1}<5 \mathcal{M}_{\odot}\right)$ binaries suggests a dependence of the mass-ratio distribution on the mass of the primary: $f\left(q, \mathcal{M}_{1}\right)$.

By combining the photometric information with orbital elements constraints, we have derived the mass-ratio distribution for intermediate-mass stars. As is the case for low-mass stars, this distribution does not have any maximum close to $\mathcal{M}_{2} / \mathcal{M}_{1}=1$.
\end{abstract}

\section{INTRODUCTION}

An important part of the observational effort done with the CORAVEL spectrometers (Baranne et al. 1979) is devoted to systematic surveys related to binary stars. The ultimate aim of these surveys is to determine the distributions of orbital elements for several unbiased samples of stars of different masses and ages, and belonging to different environments. Due to the stability of these spectrometers, to the time span of the observations (more than ten years for most of these surveys) and due also to the good quality of the radial-velocity measurements (about $0.3 \mathrm{~km} / \mathrm{s}$ ), we have derived well defined orbital element distributions.

Distributions of orbital elements still conserve some information on the processes of stellar formation, and on interaction processes like tidal circularization, dynamical interaction, mass transfer or wind accretion. Despite the huge efforts done in the domain of stellar duplicity, our knowledge of orbital element distributions, their dependence on the mass of the primary, on its age, and so on, are very limited. 


\section{CORAVEL SURVEYS}

Our surveys are carried in both hemisphere using the CORAVEL spectrometers mounted on the $1-\mathrm{m}$ Swiss telescope at the Haute-Provence Observatory and on the 1.54-m Danish telescope at La Silla (ESO) Observatory. Among the most important CORAVEL surveys, we can mention a sample of about $900 \mathrm{dwarf}$ stars ( $F$ to $M$ ) in the solar vicinity. This sample will give us properties of the duplicity of low-mass stars, mainly with primary masses $\mathcal{M}_{1}$ from 0.3 to 1.2 $\mathcal{M}_{\odot}$.

An extensive discussion has already been published for the G-dwarfs of the solar vicinity (Duquennoy \& Mayor 1991, later referred to as DM91). Preliminary results are given in section 3 for the K-dwarf survey. Despite the fact that K- and M-dwarfs represent more than $90 \%$ of the total number of stars in the Galaxy, little has been published on statistical properties of binaries with very low masses (see recent analyses by Fisher \& Marcy (1992) and by Tokovinin (1992)). In the same mass range, important data can be derived from red dwarfs in open clusters. Such data provide information on the time dependence of the orbital elements. An example is the evolution with time of the $(e, \log P)$ diagram as a result of the tidal dissipation (cf. a recent review by Zahn 1992). A discussion of constraints on tidal circularization derived from coeval samples of binaries has been published by Duquennoy et al. (1992a) and by Mathieu et al. (1992).

About 900 red dwarfs in the field of open clusters are monitored, of which 600 stars are real members of these systems. A wide range of ages are represented by these clusters from $10^{7}$ to $5 \times 10^{9}$ years. The dynamical mass segregation in open clusters will enhance the rate of binaries in the central part of the clusters. An unbiased statistic on binaries in clusters imply first the identification of member stars in the very external part of these systems. Efforts have been done to complete the detection of new members in the corona of Praesepe (Mermilliod et al. 1990) and Pleiades (Rosvick et al. 1992). A study of duplicity in the F5-K0 spectral range for the Pleiades has been published (Mermilliod et al. 1992).

The percentage of short period binaries $(P<1000$ days $)$ in the Pleiades is close to $13 \%$ in the core but probably less than $10 \%$ when we consider the whole cluster. The mass-ratio distribution of solar-type binaries in the Pleiades is flat in the interval $0.5<\mathcal{M}_{2} / \mathcal{M}_{1}<1$. No maximum of the mass-ratio distribution is visible close to 1 . Contrary to some existing claim in litterature, SB2 stars with similar components are not particularly abundant in young open clusters (at least for $\mathcal{M}_{1}$ close to one solar mass). Our samples of K-giants in open clusters and supergiants allow the determination of orbital element distributions for stars heavier than two solar masses.

The combined sample of G- and K-dwarfs in the solar vicinity reveals a lot of very low amplitude binaries. Their frequency is large enough to indicate the existence of brown dwarf companions (Section 4). The distribution of orbital elements for K-giants with masses in the range of 2 to 5 solar masses have been compared to low-mass binaries in clusters and in the field. Such comparison permits a direct test of a possible variation of the mass-ratio distribution when the primary mass increases (Section 5). A discussion of the combined photometric properties and orbital elements for intermediate-mass giants allows a direct determination of the mass-ratio distribution (Section 6). 

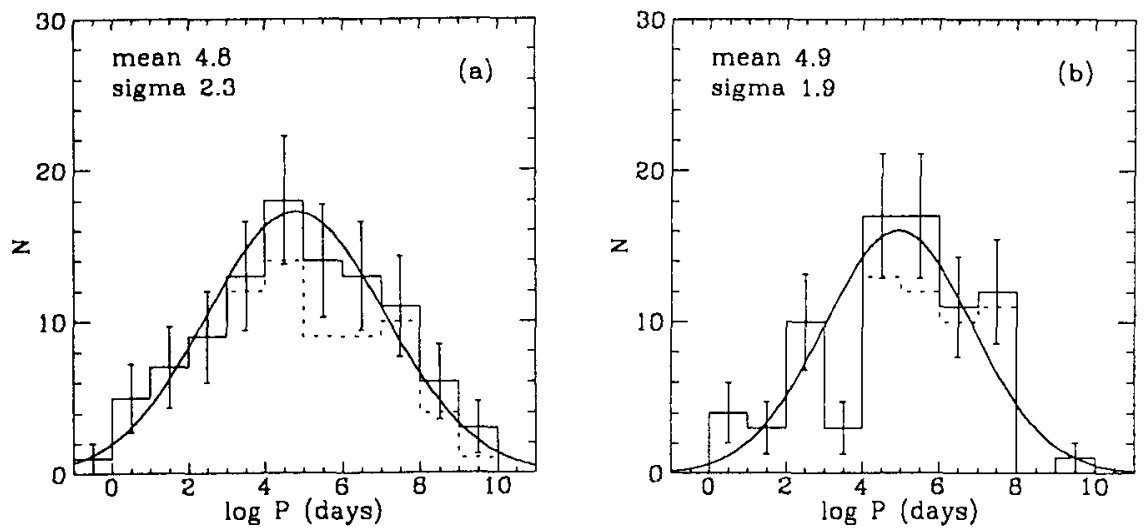

FIGURE 1. Period distributions for nearby $G(a)$ and $K(b)$ dwarfs, with (continuous) and without (dashed) detection biases taken into account. The mean and sigma of the fitting gaussian curves are indicated.

\section{AN UNBIASED SURVEY OF NEARBY K-DWARF STARS}

In parallel to the G-dwarf survey discussed by DM91, a similar survey has been carried out since 1983 for the K-dwarfs of the Gliese's (1969) catalogue. An unbiased sample, selected by the trigonometric parallax only, includes all Kdwarfs (luminosity classes IV to VI), with a declination north of $-10^{\circ}$ and a parallax larger than $0^{\prime \prime} .0455$. A first comparison can be made with the G-dwarf sample (Table 1):

TABLE 1. Comparison of nearby K- and G-dwarf star samples studied with CORAVEL

\begin{tabular}{|lcl|}
\hline Sample & K-dwarfs & G-dwarfs \\
\hline Number of primaries & 172 & 164 \\
Spectroscopic binaries & 19 & 24 \\
Visual binaries & 15 & $27(+5)$ \\
Common proper motion pairs & $33(+11)$ & $29(+14)$ \\
\hline
\end{tabular}

The values given in parenthesis correspond to bias corrections. It seems that the fraction of binaries is slightly smaller among K-dwarfs than for solar type stars. Such an effect has also been found by Tokovinin (1992) and Fischer \& Marcy (1992) in smaller samples of $\mathrm{K}$ and $\mathrm{M}$ dwarfs. The gaussian distribution of $\log P$ observed for the G-dwarfs (DM91) is also found among $\mathrm{K}$-dwarfs, with a similar mean period (Figure 1).

No significant differences appear when we compare the $(e, \log P)$ distributions for $\mathrm{K}$ and $\mathrm{G}$ binaries. When we add the two samples, a cut-off period 


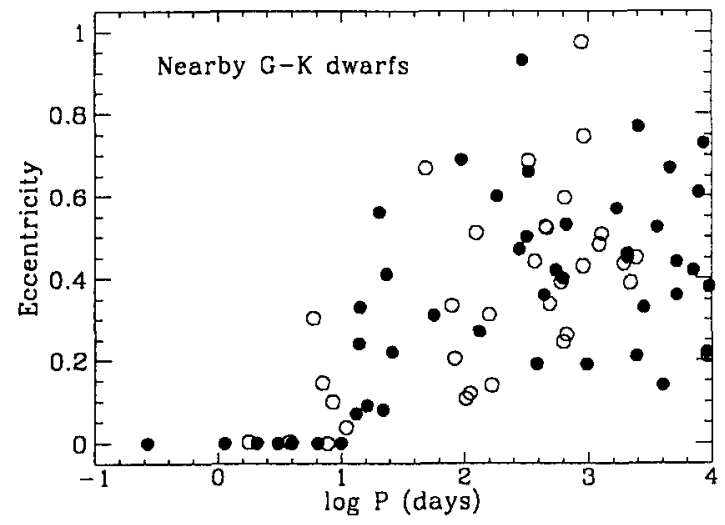

FIGURE 2. The $(e, \log P)$ diagram for the combined sample of nearby $G$ (filled circles) and $K$ (open circles) dwarfs.

due to tidal circularization appears around 6-10 days (Figure 2). However, due to the decrease of the period during the circularization process, the largest period with circular orbit seems more appropriate to characterize the cut-off. (Duquennoy et al. 1992a).

Apart from the conspicuous tidal circularization effect for short-period systems, take note of the well defined upper envelope and also of the presence of a very exceptional system close to $\log P(d)=3$, with an eccentricity of $0.9752 \pm 0.0003$ (Duquennoy et al. $1992 \mathrm{~b}$ ), the most eccentric spectroscopic system known to date. But probably a most significant constraint for the theory of binary formation is the absence of any circular orbits (above the tidal circularization cut-off). The cumulative distribution of eccentricities for SBs $(\log P(d)>1.5)$ indicates less than $5 \%$ of orbits with an eccentricity less than 0.15. Stellar formation processes do not create binaries with circular orbits. Such a property has been found for primary masses $\mathcal{M}_{1}=0.6$ to $5 \mathcal{M}_{\odot}$. An extensive discussion of the K-dwarf survey is in preparation.

\section{DO COMPANIONS WITH $\mathcal{M}_{2}<0.08 \mathcal{M}_{\odot}$ EXIST AMONG G AND K DWARF BINARIES?}

If we consider all the G- and K-dwarfs monitored with the CORAVEL spectrometers, and if we relax the severe constraint due to the parallax limit (the Branch effect is not important for this discussion), we have a combined sample of 540 primaries. Among them 62 spectroscopic binaries have a period less than 3000 days and 9 spectroscopic binaries have a lower limit for the mass of the companion less than $0.08 \mathcal{M}_{\odot}$. Of course a fraction of these 9 stars have a very low $\mathcal{M}_{\mathbf{2}} \sin i$ (derived from the spectroscopic mass function) only due to a small value of $\sin i$. In the following we assume a random distribution of the orbital inclinations $i$. 

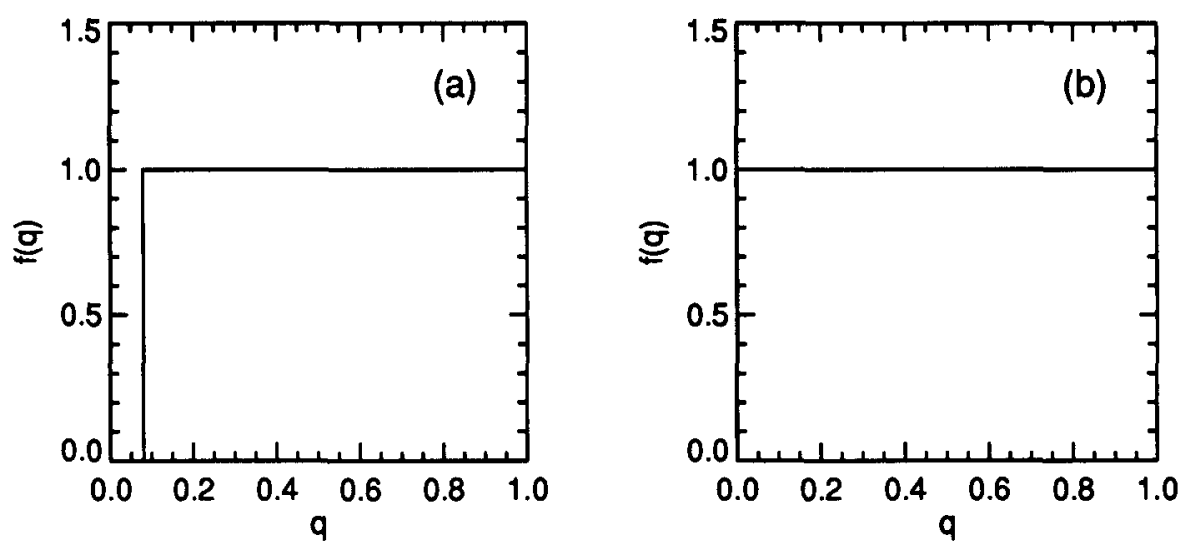

FIGURE 3. Simple models of mass-ratio distributions tested with the 9 observed low mass-function nearby G-K dwarf binaries, assuming (a) absence, (b) existence of brown dwarf companions.

i) We have first tried to estimate if these 9 stars with $\mathcal{M}_{2} \sin i<0.08 \mathcal{M}_{\odot}$ can result from a mass-ratio distribution without brown dwarfs (Figure 3a): we have assumed a flat distribution of $f(q)$ from $q=0.08$ to $q=1$, where $q=\mathcal{M}_{2} / \mathcal{M}_{1}$ is the mass-ratio.

Such a distribution is a very reasonable guess when Binary stars with companions less massive than $\mathcal{M}_{2}=0.08 \mathcal{M}_{\odot}$ exist. We consider the analysis of the short period solar-type binaries (Mazeh et al. 1992). We have only a $2 \%$ probability to observe as many as 9 stars with $\mathcal{M}_{2} \sin i<0.08 \mathcal{M}_{\odot}$. When we take into account the detection bias, especially important for binaries with very low mass companions, the probability to observe these 9 stars by chance is only $0.026 \%$ !

ii) A second statistical analysis has been done using a flat $f(q)$ distribution (Figure $3 b$ ), i.e. a uniform distribution from $q=0$ to $q=1$. Once again, taking into account the detection bias, we have a probability less than $10 \%$ to observe 9 stars or more with $\mathcal{M}_{2} \sin i<0.08 \mathcal{M}_{\odot}$. The flat extrapolation of $f(q)$ below $0.08 \mathcal{M}_{\odot}$, towards $\mathcal{M}_{2}=0$ is probably a lower limit to the real mass-ratio distribution.

\section{IS THE MASS-RATIO DISTRIBUTION $F(m) / \mathcal{M}_{1}$ ALSO A FUNCTION OF THE PRIMARY MASS $\left(\mathcal{M}_{1}\right)$ ?}

Apart from the $\sin i$ term, the relative mass function

$$
\frac{f(m)}{\mathcal{M}_{1}}=\frac{q^{3} \sin ^{3} i}{(1+q)^{2}}
$$




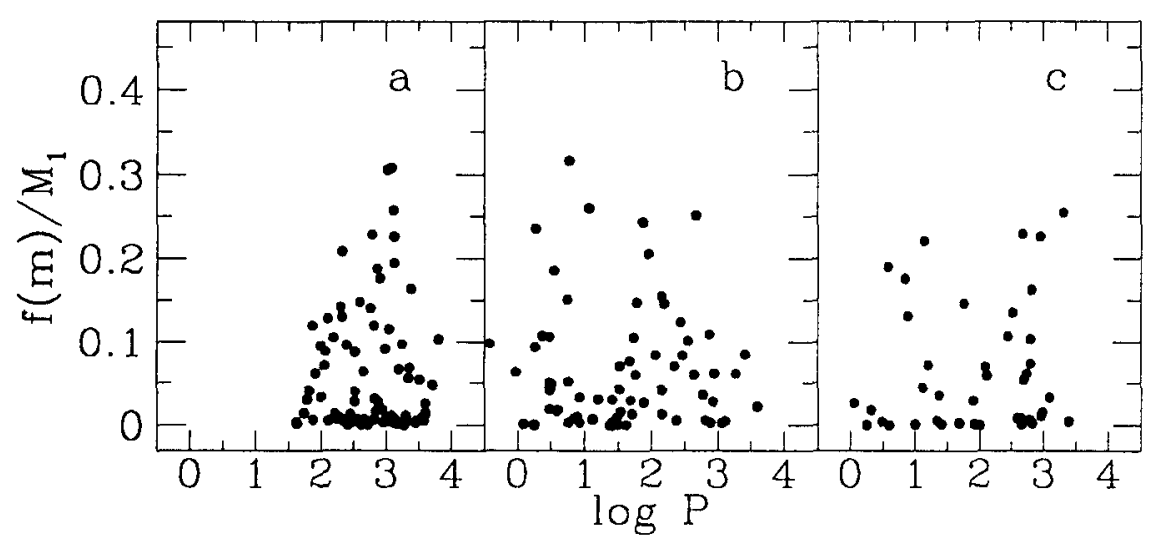

FIGURE 4. Distribution of the relative mass function $f(m) / \mathcal{M}_{1}$ for three samples: (a) cluster red giants, (b) cluster dwarfs, (c) G-K nearby dwarfs

is a quantity depending only on the mass-ratio distribution $f(q)$. Let us compare the distributions of $f(m) / \mathcal{M}_{1}$ vs $\log P$ for different (unbiased) samples of selected primary masses $\mathcal{M}_{1}$.

From a sample of 663 red giant stars members of open clusters, we have detected 152 spectroscopic binaries and determined 87 orbits. Among these binaries, 61 have masses between 2 and $5 \mathcal{M}_{\odot}$ (determined by the isochrone fitted to the parent cluster colour-magnitude diagram). Two low-mass star samples can be used for comparison, with primary masses between 0.7 and 1.3 $\mathcal{M}_{\odot}$ :

- the F, G, K dwarfs in open clusters (Hyades, Pleiades, Praesepe, Coma Berenices and $\alpha$ Persei).

- the late $\mathrm{F}, \mathrm{G}$ and $\mathrm{K}$ dwarfs in the solar vicinity.

Figure 4 illustrates the $f(m) / \mathcal{M}_{1}$ dependence on $\log P$ for the three above mentioned samples. The red giant sample $\left(\overline{\mathcal{M}_{1}}=2.7 \mathcal{M}_{\odot}\right)$ presents an excess of companions (compared to dwarfs with $\overline{\mathcal{M}_{1}}=1 \mathcal{M}_{\odot}$ ) with $f(m) / \mathcal{M}_{1}<0.015$ or $\mathcal{M}_{2}<0.8 \mathcal{M}_{\odot}$. Such an excess of companions with mass $\mathcal{M}_{2}<0.8 \mathcal{M}_{\odot}$ could result either from white dwarfs or low-mass main sequence stars. In case of degenerate companions resulting from the past evolution of the more massive original primary, we should observe a difference between the $(e, \log P)$ diagrams for stars with $f(m) / \mathcal{M}_{1}<0.01$ or with $f(m) / \mathcal{M}_{1}>0.02$. Such a difference is a result of the wind accretion (see Boffin \& Jorissen (1989), and the study of S stars by Jorissen \& Mayor (1992)). No difference is observed when we compare the $(e, \log P)$ diagrams for samples with different values of the ratio $f(m) / \mathcal{M}_{1}$. We then conclude that the excess of companions with $\mathcal{M}_{2}<0.8$ $\mathcal{M}_{\odot}$ is most probably due to low-mass main sequence secondaries. The massratio distribution possibly depends on the mass of the primary. 


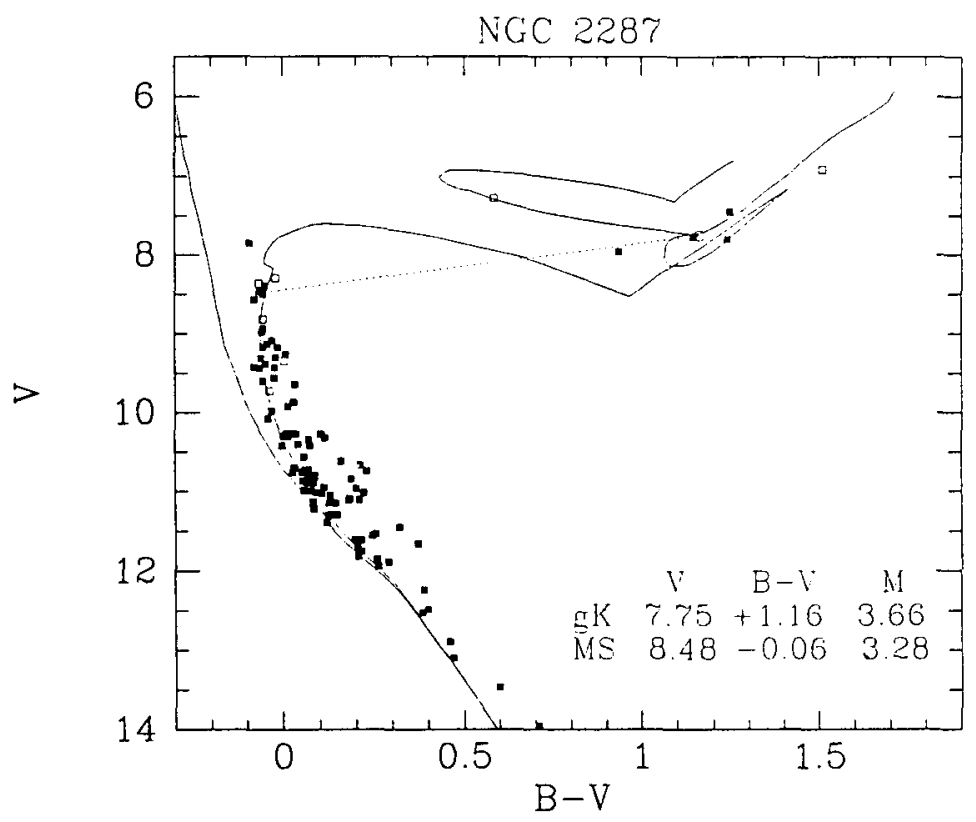

FIGURE 5. Photometric separation of a composite binary. The drawn curves are the ZAMS, an isochrone $(\log t=8.38)$ and the binary loop. The dashed line connects the positions of the primary and secondary.

\section{MASS-RATIO DISTRIBUTION FOR INTERMEDIATE- MASS STARS $\left(2 \mathcal{M}_{\odot}<\mathcal{M}_{1}<5 \mathcal{M}_{\odot}\right)$}

Many spectroscopic binaries appear in the Hertzsprung gap as a result of the addition of the fluxes of a red giant primary and a main sequence secondary. A photometric analysis of spectroscopic binaries in the gap gives $\mathcal{M}_{1}$ and $\mathcal{M}_{2}$, and the mass-ratio $\mathcal{M}_{2} / \mathcal{M}_{1}$. Figure 5 illustrates such an example of photometric separation of a composite binary located within the Hertzsprung gap of the open cluster NGC 2287.

For spectroscopic binaries located on the giant branch or in the clump, the absence of any photometric effect gives an upper limit to the mass of the companion $\mathcal{M}_{2, \max }$ (and $q_{\max }$ ). The mass function derived from the spectroscopic orbit allows an estimation of a lower limit to the mass of the secondary, $\mathcal{M}_{2, \text { inf }}$ and $q_{\min }$. Most of the time $q_{\min }$ is close to $q_{\max }$, and a well determined mass-ratio function $f(q)$ can be built. The mass-ratio function for binary stars of period less than 3000 days and primaries in the domain of 2 to $5 \mathcal{M}_{\odot}$ is shown in Figure 6. Such a distribution is not significant below $q=0.2$, due to uncorrected and important detection biases in this range.

But once again, the mass-ratio function appears to be almost flat for $q>$ 0.4 , as already established for solar type stars in the field (Mazeh et al. 1992, Tokovinin 1992) and in open clusters, (cf. the study of the Pleiades, Mermilliod 


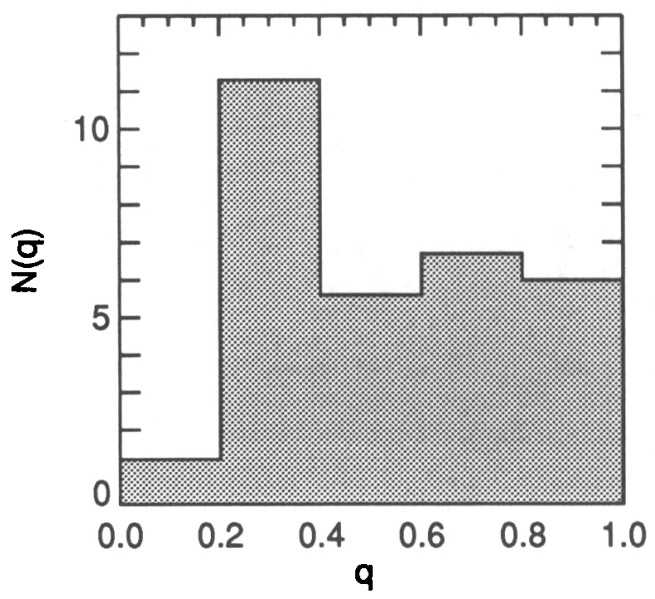

FIGURE 6. Mass-ratio distribution of red giant spectroscopic binaries with a primary mass in the range $2<\mathcal{M}_{1}<3 \mathcal{M}_{\odot}$

et al. 1992, Figure 6). The mass-ratio distribution $f(q)$ does not present a peak close to $q=1$. This result is valid for primary mass $\mathcal{M}_{1}$ in the interval 0.7 $\mathcal{M}_{\odot}<\mathcal{M}_{1}<5 \mathcal{M}_{\odot}$.

\section{REFERENCES}

Baranne, A., Mayor, M., \& Poncet, J.-L. 1979, Vistas Astron., 23, 279

Boffin, H. \& Jorissen, A. 1988, $A \& A, 205,155$

Duquennoy, A. \& Mayor, M. 1991, $A \& A, 248,485$

Duquennoy, A., Mayor, M., \& Mermilliod, J.-C. 1992a Binaries as Tracers of Stellar Formation, eds A. Duquennoy and M. Mayor, Cambridge, in press

Duquennoy, A., Mayor, M., Andersen, J., Carquillat, J.-M., \& North, P. 1992b, A 8 A Letters, 254, L13

Fisher, D. \& Marcy, G. 1992, $A p J$, in press

Gliese, W. 1969, Veröff. Astron. Rechen Inst. Heidelberg, No 22

Jorissen, A. \& Mayor, M. 1992, $A \& A$, in press

Mazeh, T., Goldberg, D., Duquennoy, A., \& Mayor, M. 1992, ApJ, in press

Mathieu, R.D., Duquennoy, A., Latham, D.W., Mayor, M., Mazeh, T., \& Mermilliod, J.-C. 1992, Binaries as Tracers of Stellar Formation, eds A. Duquennoy and M. Mayor, Cambridge, in press

Mermilliod, J.-C., Duquennoy, A., \& Mayor, M. 1990, $A \& A, 235,114$

Mermilliod, J.-C., Rosvick, J., Duquennoy, A., \& Mayor, M. 1992, A\&A, submitted

Rosvick, J., Mermilliod, J.-C., \& Mayor, M. 1992, $A \& A, 255,130$

Tokovinin, A. 1992, A\&A, 256, 121

Zahn, J.-P. 1992, Binaries as Tracers of Stellar Formation, eds A. Duquennoy and M. Mayor, Cambridge, in press 


\section{DISCUSSION}

ABT: Do you care to comment on possible differences between high- and low-velocity stars?

MAYOR: In a limited analysis of halo stars, carried out a few years ago with G. Yarniewicz, we obtained the same binary rate as for population I stars. But, the much larger study carried out at CfA on halo stars will significantly improve our knowledge of the orbital characteristics of halo binaries. It is still my perception that the distributions for populations I and II are quite similar.

VAN DESSEL: You have an observing program both in France and at ESO. I would think that the quality of the sky is better at ESO. Does that improve the quality of your results?

MAYOR: No, the radial velocity uncertainties are essentially the same in both hemispheres. The only difference is that the limiting magnitude, is somewhat better at ESO. 